Research paper

\title{
Leopardus wiedii Papillomavirus type 1, a novel papillomavirus species in the tree ocelot, suggests Felidae Lambdapapillomavirus polyphyletic origin and host-independent evolution
}

\author{
Gaby Dolz ${ }^{\mathrm{a}, 1}$, Roberta Lecis ${ }^{\mathrm{c}, 1}$, Antony Solórzano-Morales ${ }^{\mathrm{a}}$, Fernando Aguilar-Vargas ${ }^{\mathrm{a}}$, \\ Tamara Solórzano-Scott ${ }^{\mathrm{a}}$, Roberto Peña ${ }^{\mathrm{a}}$, Rosanna Zobba ${ }^{\mathrm{c}}$, Gessica Tore ${ }^{\mathrm{c}}$, Marco Pittau ${ }^{\mathrm{b}, \mathrm{c}}$, \\ Alberto Alberti ${ }^{\mathrm{b}, \mathrm{c}, *}$

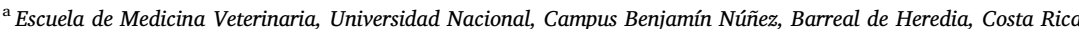 \\ ${ }^{\mathrm{b}}$ Mediterranean Center for Disease Control, University of Sassari, 07100 Sassari, Italy \\ ${ }^{\mathrm{c}}$ Department of Veterinary Medicine, University of Sassari, Via Vienna 2, 07100 Sassari, Italy
}

\section{A R T I C L E I N F O}

\section{Keywords:}

Animal papillomavirus

Felidae infectious diseases

Skin microbiome

PV evolution

Margay

Viral taxonomy

\begin{abstract}
A B S T R A C T
The limited knowledge on Papillomavirus diversity (particularly in wild animal species) influences the accuracy of PVs phylogeny and their evolutionary history, and hinders the comprehension of PVs pathogenicity, especially the mechanism of virus - related cancer progression. This study reports the identification of Leopardus wiedii Papillomavirus type 1 (LwiePV1), the first PV type within Lambdapapillomavirus in a Leopardus host. LwiePV1 full genome sequencing allowed the investigation of its taxonomic position and phylogeny. Based on results, LwiePV1 should be assigned to a novel PV species providing evidence for a polyphyletic origin of feline lambda PVs, and representing an exception to codivergence between feline lambda PVs and their hosts. Results improve our knowledge on PV diversity and pave the way to future studies investigating biological and evolutionary features of animal PVs.
\end{abstract}

\section{Introduction}

Papillomaviruses (PVs) are an ancient and well-populated family of small non-enveloped viruses infecting a large assortment of vertebrate species; they have been rescued from fishes, reptiles, birds and mammals, but they have not yet been found in amphibians (Antonsson and McMillan, 2006; Bernard et al., 2010; Rector and Van Ranst, 2013; López-Bueno et al., 2016). PVs are DNA viruses with a double-stranded, circular, covalently closed genome of approximately $8 \mathrm{~KB}$ in size. They are generally species specific and induce proliferations of stratified squamous epithelia of skin and mucosal tissue. With regard to their pathogenicity, PVs range from non-pathogenic to highly pathogenic, and cause the onset of variable clinical signs, such as papillomas, fibropapillomas, and cancer (Van Ranst et al., 1992; De Villiers et al., 2004; Zur Hausen, 2009). At present more than 220 different types of human PVs (HPVs) belonging to 5 genera (Alpha, Beta, Gamma, $\mathrm{Mu}, \mathrm{Nu}$ ) have been identified, containing at least 41 different species (Rector and Van Ranst, 2013; Tore et al., 2017). Although PVs have been isolated from different vertebrate species (Rector and Van Ranst, 2013;
Alberti et al., 2010; Lecis et al., 2014; Scagliarini et al., 2013; Tore et al., 2017), global animal PV diversity does not reach up the one found in human (Rector and Van Ranst, 2013; Lecis et al., 2014). As a consequence, the majority of PVs diversity in animals still has to be investigated and uncovered.

To date, according to the papillomavirus episteme, more than $70 \mathrm{PV}$ species have been detected in approximately 72 vertebrate host species, and classified in 46 PV genera (Van Doorslaer et al., 2013). Beside mammals, PV has been found in fishes (López-Bueno et al., 2016), birds (Tachezy et al., 2002; Terai et al., 2002; Van Doorslaer et al., 2009; Varsani et al., 2014), and reptiles (Herbst et al., 2009; Lange et al., 2011). In animals, the most studied PVs infections are those of domestic hosts (Lecis et al., 2014; Tore et al., 2017; Lange et al., 2013), with 23 PV types identified in cattle (Zhu et al., 2012; Da Silva et al., 2016), 20 in dogs (Van Doorslaer et al., 2013), 8 in horses (Lange et al., 2013), 5 in cats (Munday et al., 2013; Munday, 2014; Munday et al., 2017), and 4 in sheep (Alberti et al., 2010; Tore et al., 2017). Also, the presence of $\mathrm{PV}$ is increasingly being investigated in wildlife hosts, such as the bottlenose dolphin (7 PV species), the giant panda (4 PV species), and

\footnotetext{
* Corresponding author at: Mediterranean Center for Disease Control, University of Sassari, 07100 Sassari, Italy

E-mail address: alberti@uniss.it (A. Alberti).

${ }^{1}$ These authors contributed equally to this work.
} 
the Caribbean manatee (4 PV species).

So far, $4 \mathrm{PV}$ species have been identified and fully sequenced in wild Felidae Rector et al., 2007: the bobcat LrPV1, the puma PcPV1, the Asiatic lion PlpPV1, the snow leopard UuPV1. Moreover 5 PV species (FcaPV1 to 5) have been rescued from domestic cat (Munday et al., 2013; Munday et al., 2017; Lange et al., 2009a).

With the exception of FcaPV2 (Dyotethapapillomavirus), and FcaPV3 to 5 (Taupapillomavirus), Felidae PVs belong to the Lambdapapillomavirus genus, together with the Canidae CPV1 and CPV6 (Isegawa et al., 1995; Lange et al., 2009b), the Procyonidae PlPV1 (Rector et al., 2005), the Mustelidae ElPV1 (unpublished), the Ursidae AmPV4 (Zhang et al., 2017), and the Hyenidae CcrPV1 (Stevens et al., 2013).

Phylogenetic analysis of lambdapapillomaviruses demonstrated that the evolutionary history of Felidae PVs mirrors that of their feline hosts, providing evidence for long-term virus-host co-speciation of feline PVs and also providing an estimate of Lambdapapillomavirus evolutionary rate by applying host-fossil calibrations (Rector et al., 2007).

Here we report the full genome sequence and characterization of LwiePV1, the first Lambdapapillomavirus identified in a Leopardus species, the tree ocelot Leopardus wiedii. The tree ocelot (or margay) is a small Neotropical wild felid native to Central and South America, listed as Near Threatened on the IUCN Red List. The species, solitary and nocturnal, lives in evergreen and deciduous forests, is predominantly uncommon to rare throughout its range and appears genetically diverse across its habitat (De Oliveira et al., 2015).

LwiePV1 genomic features, taxonomy, and evolutionary history are investigated. Evidence for polyphyletic origin of Felidae Lambdapapillomavirus and exception to host-dependent evolution are discussed.

\section{Material and methods}

\subsection{Clinical case and samples}

During a survey conducted to detect and identify cutaneous PVs in different species of Costa Rica wildlife, a female tree ocelot (see supplementary material), aged 7.5 years and hosted in the Catarata de la Paz Center in Poàs, Alajuela, was hospitalised in the Veterinary Clinic of the "Escuela de Medicina Veterinaria, Heredia, Costa Rica", to perform a routinary ultrasound and to take blood samples. Although the feline did not show any skin lesion, cutaneous swabs were taken from the area surrounding the feline ears and kept at $4{ }^{\circ} \mathrm{C}$ for subsequent DNA extraction. The animal died one year after returning to the Catarata de la Paz Center, due to severe respiratory symptoms unrelated to Papillomavirus. DNA was extracted from swabs using the QiAmp DNA Mini Kit (Qiagen), following manufacturer recommendations, and stored at $-20^{\circ} \mathrm{C}$. Any potential case of environmental contamination or contact transmission during hospitalisation was ruled out as the margay did not have any contact with other cats or wildlife during hospitalisation. Moreover, samples from another margay living in a geographically distinct region of Costa Rica tested positive to this novel virus (see supplementary material).

All methods were performed in accordance with the relevant guidelines and regulations according to Costa Rica low following an ethical approval from the National System of Conservation Areas (SINAC, Ministry of Environment and Energy of Costa Rica, investigation permit: ACOPACC-GASP-INV-024-16).

\subsection{PV genome investigation}

To detect PV presence and identify PV genomes, traditional PCR based on a conserved fragment of L1 gene, using primers FAP59/FAP64 (targeting $480 \mathrm{bp}$ of the L1 gene) was performed, as well as the rolling circle amplification (RCA) technique combined with restriction enzyme digestion.
FAP PCR amplifications were performed in a $50 \mu$ volume containing approximately $150 \mathrm{ng}$ of DNA, and by following Qiagen recommendations for Taq polymerase. PCR profile included an initial denaturation step at $94^{\circ} \mathrm{C}$ for $10 \mathrm{~min}$, followed by 40 cycles of denaturation $(1 \mathrm{~min})$, annealing at $50{ }^{\circ} \mathrm{C}(1 \mathrm{~min})$ and extension at $72{ }^{\circ} \mathrm{C}$ (1 min), and a final extension at $72{ }^{\circ} \mathrm{C}$ for $2 \mathrm{~min}$.

RCA was performed by using the TempliPhi 100 Amplification Kit (GE Healthcare), according to previously described protocols (Alberti et al., 2010). Shortly, extracted DNA samples were mixed with $10 \mu \mathrm{l}$ of sample buffer and heated for $3 \mathrm{~min}$ at $95{ }^{\circ} \mathrm{C}$. Samples were transferred on ice and mixed with $0.4 \mu \mathrm{l}$ of TempliPhi enzyme mix, TempliPhi reaction buffer $(10 \mu \mathrm{l}), 0.4 \mu \mathrm{l}$ of $10 \mathrm{mM}$ dNTPs, and random hexamers. RCA reactions were incubated for $16 \mathrm{~h}$ at $30{ }^{\circ} \mathrm{C}$, and polymerase was inactivated at $65{ }^{\circ} \mathrm{C}$ for $10 \mathrm{~min}$. RCA products were digested with SacI, BamHI, and EcoRI, and run on a $0.8 \%$ agarose gel to visualize a band (or bands) consistent with the length of a papillomaviral genome.

\subsection{Primers design, long template PCR and sequencing}

Based on FAP59/FAP64 product sequencing, primer pairs LwiPV1F/ LwiPV1R (5'-AAGCTGGATGACACTGG-3', 5'-GGTTTGGAGAAACCTTA GGC-3'), and LwiPV1NESTF/LwiPV1NESTR (5'-CATAGCAATGGATCC GAAGC-3', 5'-CCATTATCACCAGGCTGTTG-3'), were newly designed, and combined to amplify the entire viral genome by long template nested PCR. Long template high-fidelity PCR amplifications were performed using AccuTaq LA DNA Polymerase (Sigma-Aldrich) on RCA products.

Both initial and nested amplifications were performed (with the appropriate primers) in a $50 \mu \mathrm{l}$ volume containing approximately $200 \mathrm{ng}$ of template DNA, $0.5 \mathrm{mM}$ of each dNTPs, $0.4 \mu \mathrm{M}$ each of Forward and Reverse primers, $1 \times$ AccuTaq LA Buffer and AccuTaq PCR enzyme DNA polymerase, according to manufacturer's protocol. Reaction profiles were: $30 \mathrm{~s}$ at $98{ }^{\circ} \mathrm{C}$, followed by 30 cycles of denaturation at $94{ }^{\circ} \mathrm{C}$ for $30 \mathrm{~s}$, annealing at $60^{\circ} \mathrm{C}$ for $20 \mathrm{~s}$, and extension at $68^{\circ} \mathrm{C}$ for $10 \mathrm{~min}$, with a final extension step at $68^{\circ} \mathrm{C}$ for another $10 \mathrm{~min}$.

The complete genome sequence of Leopardus wiedii Papillomavirus type 1 (LwiePV1) was obtained by combining sequencing of FAP product with sequencing of long template nested PCR. Long template nested PCR product was sequenced by primer-walking with five sets of newly designed specific primers (see supplementary material). Before sequencing PCR products were purified using DNA Clean \& Concentrator purification kit (Zymo Research, CA, USA). All products were directly sequenced in both forward and reverse directions by BMR Genomics Sequencing Service (Padova, Italy).

LwiePV1 genome sequence was deposited in GenBank under accession number MH910493.

Open reading frames (ORFs) were predicted using the ORF Finder tool on the NCBI server of the National Institutes of Health (https:// www.ncbi.nlm.nih.gov/orffinder/). Pairwise sequence alignments were obtained with ClustalW(Thompson et al., 1994) and MUSCLE (http:// www.ebi.ac.uk/Tools/msa/muscle/) and sequences pairwise percent identities were investigated by using MUSCLE and the Bioedit (Hall, 1999) identity matrix options. The compute pI/MW tool (http://www. expasy.org/tools/pitool.html) was used to calculate molecular weight of putative proteins.

\subsection{Phylogenetic analyses, time trees, and tree topology tests}

The nucleotide sequences of LwiePV1 and 108 other PVs (see supplementary material) were imported in DAMBE version 4.2.7 (Xia and Xie, 2001) and aligned at the amino acid level with ClustalW. Then, nucleotide alignments were obtained by using the aligned amino acid sequences as an alignment guide. This approach was followed independently for the E1, E2, and L1 ORFs, and according to Gottschling and cowokers (Gottschling et al., 2011), a compiled alignment was obtained by pasting together the 3 orfs. The $\mathrm{E} 4$ region nested in the E2 
gene was not considered in the molecular analyses.

The Maximum Likelihood method based on the General time reversible (GTR) model (Le and Gascuel, 2008) was used to reconstruct the evolutionary history of LwiePV1 and the 108 PVs considered in this study. This method was identified among 24 different nucleotide substitution models as the best-suited evolutionary model for our data by using the: "find best DNA/protein Models" option of MEGA6 (Tamura et al., 2013), and by selecting the model with the lowest BIC score (Bayesian Information Criterion), considered to describe the substitution pattern the best. Trees for the heuristic search were calculated by applying Neighbor-Joining to a matrix of pairwise distances, estimated using the Maximum Composite Likelihood (MCL) approach. Among sites evolutionary rate differences where modelled by using a discrete Gamma distribution ( 5 categories $(+\mathrm{G}$, parameter $=1.1748)$ ).

Evolutionary tests were conducted in MEGA6 (Tamura et al., 2013) by including 109 nucleotide sequences in the analysis. A total of 3033 positions were included in the final dataset, while positions containing missing data or gaps were eliminated. Bootstrapping (Felsenstein, 1985) (1000 iterations) was used to evaluate statistical support of internal branches of the. MEGA6 was also used to scale to time phylogenetic trees, and branch lengths were calibrated according to UuPV1 and PlpPV1 divergence time, calculated considering an overall evolutionary rate of the feline PV viral coding genomes of $1.95 \times 10^{-8}(95 \%$ confidence interval $1.32 \times 10^{-8}$ to $2.47 \times 10^{-8}$ ) nucleotide substitutions per site per year (Rector et al., 2007). Branch lengths calibrations were based on relative times obtained with MEGA. Trees were edited with NJplot (Perrière and Gouy, 1996).

Host- virus co-evolutionary relationships were reconstructed using Dendroscope v.3 (Huson and Scornavacca, 2012). Papillomaviruses Newick input file was generated by exporting a subtree of the general PVs tree originally including 109 PVs. Host phylogram was generated by aligning the margay full mitochondrial genome sequence (Leopardus wiedii, KP202289) to the mitochondrial genomes of hyena (Crocuta crocuta, NC_020670), domestic dog (Canis lupus familiaris, KF907307), domestic cat (Felis catus, NC001700), bobcat (Lynx rufus, NC014456), asiatic lion (Panthera leo persica, KC834784), snow leopard (Uncia uncia, NC010638), cougar (Puma concolor, JN999997), giant panda (Ailuropoda melanoleuca, EF212882), raccoon (Procyon lotor, NC_009126), sea Otter (Enhydra lutris, AB291077), European mole (Talpa europaea, NC_002391). Host ML trees were constructed in MEGA by using the GTR + G model, calculated as the best fitting model with the same software. Virus-host coevolution was further investigated with IQtree v 1.6.10 (Nguyen et al., 2015). Briefly, nucleotide alignment of taxa included in the Felidae lambdapapillomavirus group (PlpPV1, CcrPV1, LrPV1, LwiePV1, UuPV1, PcPV1, FcaPV1), and of those basal to the same clade (CPV1, CPV6, ElPV1, PlPV1, AmPV4, TePV1), was extrapolated from the original global PVs alignment. Gaps shared by all OTUs were manually excluded from the alignment. The alignment was used to generate Maximum likelihood (unconstrained) trees based on the GTR + F + I + G4 model, identified as the best-fit model according to BIC, by using the model finder option of IQtree (Kalyaanamoorthy et al., 2017). Constrained trees were also generated by forcing LwiePV1 to join the feline lambdapapillomavirus monophyletic clade, therefore mirroring the feline hosts evolutionary history. In order to evaluate tree statistical support, and to formally accept or reject the corresponding phylogenies, 5 tree topology tests were applied to both unconstrained and constrained trees. Specifically, bootstrap proportion using RELL method test (Kishino et al., 1990; Expected Likelihood Weight test Strimmer and Rambaut, 2002; and the $p$-value of approximately unbiased (AU) test Shimodaira, 2002 were calculated.

\section{Results}

PCR reaction based on universal primers FAP59/FAP64 conducted to detect PV presence and to identify PV genomes on DNA samples extracted from Leopardus wiedii healhy skin, generated an amplicon of
$480 \mathrm{bp}$, consistent with the expected size. Bidirectional direct sequencing of the PCR products and BlastN analyses revealed an invariable L1 sequence most similar to Canine Papillomavirus type 6 (CPV6, Lambdapapillomavirus). Rolling circle amplification failed to produce a DNA band (or multiple bands) consistent with full length PV DNA ( $8 \mathrm{~kb}$ ). Nested PCR conducted on undigested RCA product with primers LwiPV1F/LwiPV1R and LwiPV1NESTF/LwiPV1NESTR resulted in a band of about $8 \mathrm{~Kb}$, potentially representing the full genome of a PV. Bidirectional direct sequencing of the $8 \mathrm{~Kb}$ amplicon by primer walking (see supplementary material for a list of primers) allowed reconstructing the entire sequence of a novel, unclassified papillomavirus, designated LwiePV1 according to the rules of current PVs nomenclature. Further PCR experiments gave evidence of circular genome of $7944 \mathrm{bp}$ in size. The complete nucleotide sequence of Leopardus wiedii Papillomavirus type 1 (LwiePV1, GenBank accession number MH910493) has a GC content of $43.7 \%$ and includes a classical noncoding region (NCR1, $411 \mathrm{bp}$ ) and the major PV ORFs: L2, L1, E1, E2, E6, and E7. Furthermore, a putative open reading frame E4 was reconstructed by combining the first few codons of E1 (nt 1048-1063) with a downstream ORF in the +2 frame of the E2 ORF (nt 3360-3943). An additional NCR (NCR2, 952 bp) between the early and late regions is also present (nt 3943-4895, Fig. 1), a signature of Lambdapapillomavirus.

LwiePV1 similarity with other lambdapapillomaviruses was defined by nucleotide and amino acid alignments. Overall, LwiePV1 shared the highest nucleotide (nt) and amino acid (aa) identities with CPV6 and CcrPV1, respectively belonging to the Lambdapapillomavirus 3 and Lambdapapillomavirus 5 species (Table 1). Following the criteria established for the classification of novel papillomaviruses, and based on the DNA sequence of the L1 gene (Bernard et al., 2010; De Villiers et al., 2004), LwiePV1 should be assigned to a novel PV species within the Lambdapapillomavirus genus.

Table 2 reports LwiePV1 main amino acid and nucleotide features. Besides transcription factor binding sites for SP1, AP1, and NF1, TATA signals and the sequence elements for PV DNA replication were found in the viral genome. In particular, $3 \mathrm{E} 2$ binding sites are present within the NCR1. Examination of the putative protein sequences reveals an ATP-dependent helicase motif located in E1 and a metal-binding motif both in E6 and E7. The canonical retinoblastoma tumour suppressor binding domain (LXCXE) is present in E7. No additional LXCXE motifs have been identified in E6. LwiePV1 LCR1 was predicted to contain 2 polyadenylation sites. A TATA box was identified at nucleotide position 334 (TATAAAAT). Three E2 binding sites (E2BS) motifs with a stringent consensus sequence (ACC-N6-GGT) were found in the LCR region, as well as an imperfect E1 binding site with a HpaI-like sequence. E1 protein contains a helicase domain with a conserved ATP-binding site [G-X(4)-GK(T/S)]. The late region encodes 2 viral capsid proteins L1 and L2. LwiePV1-L1 harbours a bipartite nuclear localization signal at C-terminus, which is likely to play a role in the nuclear translocation of L1 and L2, during the viral life cycle.

Phylogeny was inferred based on a concatenated E1/E2/L1 nucleotide sequence alignment of LwiePV1 and 108 PV-types representative of the different PV genera (Fig. 2). According to the most recent PVs classification (Scagliarini et al., 2013), resulting maximum likelihood phylogenetic trees clustered the PVs in their respective genera. Further, main genera associations were consistent with what observed by Gottschling et al. (Gottschling et al., 2011), and later studies (Lecis et al., 2014; Tore et al., 2017), and were updated with novel PV species recently discovered. In particular, 6 main genera associations can be identified (Fig. 2): 1) Eta, Teta, Dyoepsylon and Dyozeta genera, comprising birds, turtles, and bats PVs clustering in a markedly distinct branch; 2) the Artiodactyla-infecting Delta and Epsilon viruses grouping together with the PVs infecting Equidae, namely Zeta, Dyoiota, Dyorho, and the donkey Dyochi (Lecis et al., 2014) with carnivora PV of the Chi, Dyonu, Dyoeta and iota PVs forming a separate but related branch; 3) Alpha, Upsilon, Omicron, Dyodelta and Omega 

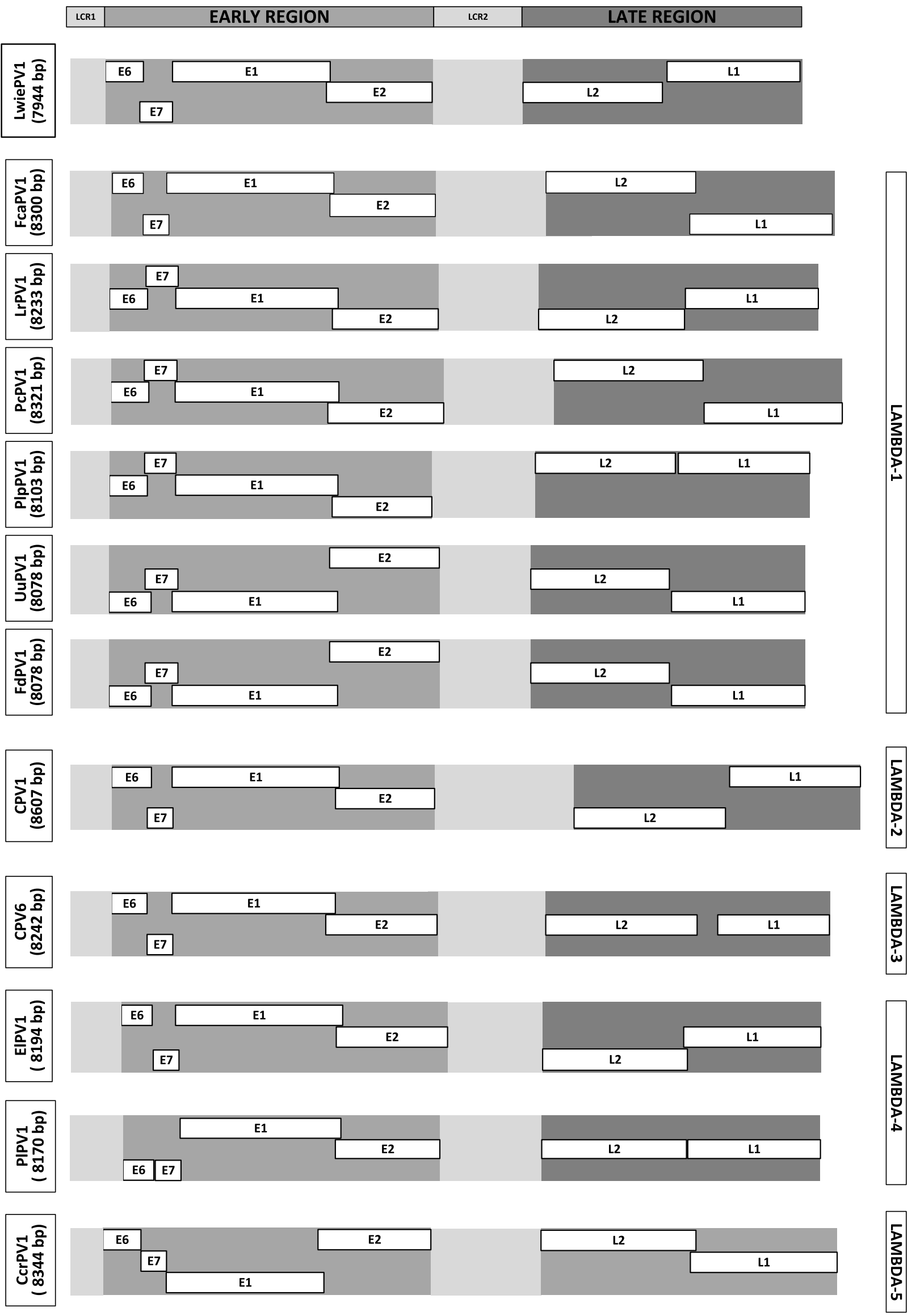

Fig. 1. General genome organisation of LwiePV1 and Lambdapapillomavirus species. 
Table 1

Nucleotide (and amino acid) identities between LwiePV1 and species belonging to Lambdapapillomavirus.

\begin{tabular}{|c|c|c|c|c|c|c|c|c|c|c|c|}
\hline \multirow{3}{*}{$\begin{array}{l}\text { PV species } \\
\text { PV type }\end{array}$} & \multicolumn{11}{|c|}{ Lambdapapillomavirus } \\
\hline & \multicolumn{5}{|l|}{ LambdaPV1 } & \multirow{2}{*}{$\begin{array}{l}\text { LambdaPV2 } \\
\text { CPV1 }\end{array}$} & \multirow{2}{*}{$\begin{array}{l}\text { LambdaPV3 } \\
\text { CPV6 }\end{array}$} & \multicolumn{2}{|l|}{ LambdaPV4 } & \multirow{2}{*}{$\begin{array}{l}\text { LambdaPV5 } \\
\text { CcrPV1 }\end{array}$} & \multirow{2}{*}{$\begin{array}{l}\text { na* }^{*} \\
\text { AmPV4 }\end{array}$} \\
\hline & UuPV1 & PlpPV1 & FcaPV1 & LrPV1 & PcPV1 & & & ElPV1 & PlPV1 & & \\
\hline E6 & $60.3(47.4)$ & $60.5(44.4)$ & $61.1(47.4)$ & $63.1(45.9)$ & $62.3(48.1)$ & $59.7(43.3)$ & $65.6(50.4)$ & $59.4(42.2)$ & $62(47.4)$ & $61.2(46.2)$ & $63.6(44)$ \\
\hline E7 & $69.5(56.0)$ & $65.9(54.8)$ & $70.6(53.8)$ & $68.3(50.5)$ & $69.7(59.1)$ & $64.0(51.1)$ & $69.2(56.3)$ & $62.2(44.6)$ & $67.4(57.6)$ & $67.2(51.6)$ & $60.9(47.8)$ \\
\hline E1 & $70.6(68.3)$ & $71.5(68.5)$ & $70.8(66.4)$ & $70.1(66.9)$ & $70.3(67.2)$ & $66.7(61.1)$ & $70.4(64.8)$ & $68.9(61.7)$ & $67.1(60.3)$ & $69.3(60.9)$ & $69.3(61.8)$ \\
\hline E2 & $64.9(55.4)$ & $64.9(56.1)$ & $64(56.7)$ & $65.3(56.6)$ & $63.7(52.9)$ & $61.7(51.1)$ & $66.1(57.8)$ & $60.1(50)$ & $61.9(51.4)$ & $61.8(49.9)$ & $63.8(53)$ \\
\hline L2 & $65.7(63)$ & $65.6(65.2)$ & $67.2(64.1)$ & $64.5(64.1)$ & $65.5(61.7)$ & $61.6(56.5)$ & $68.2(59.8)$ & $62.5(54.6)$ & $63.3(55.1)$ & $64.8(59)$ & $63.7(54.9)$ \\
\hline L1 & $68.6(71.3)$ & $69.5(70.5)$ & $69.1(72.5)$ & $70.5(70.3)$ & $69.8(71.9)$ & $68.5(67.3)$ & $71.34(71.26)$ & $69.2(66.9)$ & $66.7(66.3)$ & $69.44(68.9)$ & $68.7(65.3)$ \\
\hline
\end{tabular}

na* $=$ species not yet assigned by ICTV.

Most similar proteins are indicated in bold.

genera, including human mucosal, dolphins, the polar bear, and most giant panda viruses; 4) Nu, Sigma, and Dyokappa genera; 5) a large group including human Beta and Gamma genera together with PVs isolated from various mammals (Tau, Pi, Dyoxi, Phi, Xi, and Dyoeta); 6) Carnivora PVs of the Lambda genus related with Dyophi, Kappa, Mu, and Rho viruses.

In trees (Figs. 2, 3) Felidae PVs of the Lambda genus are included in a statistically supported monophyletic branch, to which the canine CPV6 and the hyena CcrPV1 are basal. Notably, LwiePV1 does not group with the other Felidae PVs, instead appearing in an independent Hyenidae CcrPV1 and the Canidae CPV6. Therefore, the identification of the first Lambdapapillomavirus in the genus Leopardus provides evidence for a polyphyletic origin of Felidae PVs. Viral divergence times, calculated considering an overall evolutionary rate of the feline $\mathrm{PV}$ viral coding genomes of $1.95 \times 10^{-8}\left(95 \%\right.$ confidence interval $1.32 \times 10^{-8}$ to $2.47 \times 10^{-8}$ ), indicated that LwiePV1 originated about $31.4 \mathrm{Myr}$ (24.75-46.35) ago.

Tanglegram describing co-evolutionary relationships among Felidae papillomaviruses and their hosts (Fig. 4) confirms the virus-host cospeciation observed by Rector and collegues (Rector et al., 2007) with the sole exception of LwiePV1, which falls outside the monophyletic Felidae PVs clade.

Topology statistical analyses applied to formally test the position of LwiePV1 (see material and methods) support results and provide more evidence against host virus coevolution/cospeciation (Fig. 5). Constrained trees where LwiePV1 is forced to join the Felidae lambdapapillomavirus monophyletic clade (Fig. 5B, C) are rejected by all 3 tree topology tests applied, while the position of LwiePV1 and CcrPV1 in an independent clade (Fig. 5A) in the unconstrained tree is supported by the same statistics.

\section{Discussion}

Papillomaviruses strictly coevolve with their vertebrate hosts, and evidences for host-virus codivergence have been provided for different PV genera (Rector et al., 2007; Lyal et al., 1986; Gottschling et al., 2011). However, each single host species potentially harbours uncounted PV types (see https://pave.niaid.nih.gov/, for an updated list of currently identified human and animal PV types). This is best documented for human, where over 300 different PV types have been identified, but also for animals, such as dog (20 types) and cow (23 types). Therefore it is suspected that the largest part of animal PVs diversity has yet to be uncovered (Bernard et al., 2010; Tore et al., 2017), especially in wild host species. The high diversity of PVs in single host species can not be explained by codivergence alone (Lyal et al., 1986), also considering the numerous examples of paralogous PV lineages on the same host species (Gottschling et al., 2011; Jackson, 2005). In a study conducted to quantify phylodynamic forces driving papillomavirus evolution, Gottschling and co-workers (Gottschling et al., 2011) presented a statistically supported scenario in which codivergence and alternative mechanisms, such as adaptive radiation and the realization of new ecological niches (García-Vallvé et al., 2005; Stamatakis, 2006) needed to be considered to explain the numerous PV lineages on the same host species (Jackson, 2005). Also cross-infections have been called in to explain paraphyletic or polyphyletic PVs infecting the same host (Myers et al., 1996; Chen et al., 2009).

The still limited knowledge on PV diversity (particularly in wildlife species) may influence the accuracy of PVs phylogeny. The lack of close viral relatives can eventually alter the trees topology and the "real" resolution of nodes, introducing evolutionary artefacts and altering phylogenetic inferences.

To date, a total of 5 Lambdapapilomavirus genomes have been fully

Table 2

Genomic nucleotide and amino acid features of Leopardus wiedii papillomavirus type 1 .

\begin{tabular}{|c|c|c|c|}
\hline Predicted nt-features & Position $^{\mathrm{a}}$ & Predicted aa-features & Position $^{\mathrm{a}}$ \\
\hline Genome size (bp) & 7944 & E7 Retinoblastoma tumour suppressor binding domain (LXCXE) & 841 \\
\hline GC content $(\%)$ & 43.7 & E6 Retinoblastoma tumour suppressor binding domain (LXCXE) & absent \\
\hline Sp1 binding site (GGCGGG) & 6173 & $\begin{array}{l}\text { Metal-binding motifs in E7 } \\
\text { [CXXC-X(28-30)-CXXC] }\end{array}$ & 929 \\
\hline NF1 binding sites (CGGAA) & 1508 & $\begin{array}{l}\text { Bipartite nuclear localization signal in L1 } \\
\text { KRSRLVTTSTKPRSVKRRR }\end{array}$ & 7876 \\
\hline AP1 binding sites (TGANTCA) & 7412 & $\begin{array}{l}\text { ATP-dependent helicase motive in E1 } \\
\text { [G-X(4)-GK(T/S)] }\end{array}$ & 2347 \\
\hline $\begin{array}{l}\text { TATA signals in LCR [TATAAA or TATA(A/T)A(A/ } \\
\text { T)] }\end{array}$ & 334 & Cyclin interaction motive in E1 (RXL) & absent \\
\hline E2 binding site (ACCG- ${ }_{4}$-CGGT) & $164,227,307$ & $\begin{array}{l}\text { Metal-binding motifs in E6 } \\
\text { [CXXC-X(28-30)-CXXC] }\end{array}$ & 442 \\
\hline Imperfect E1 binding site with HpaI-like sequence & 246 & & \\
\hline Polyadenylation sites ATAAA & $\begin{array}{l}231,333,2568,4051,4981,5184,6938 \\
7139,7758\end{array}$ & & \\
\hline
\end{tabular}

\footnotetext{
${ }^{\mathrm{a}}$ For both nucleotide and amino acid features, positions are numbered starting from the first nucleotide following the stop codon of the L1 ORF.
} 


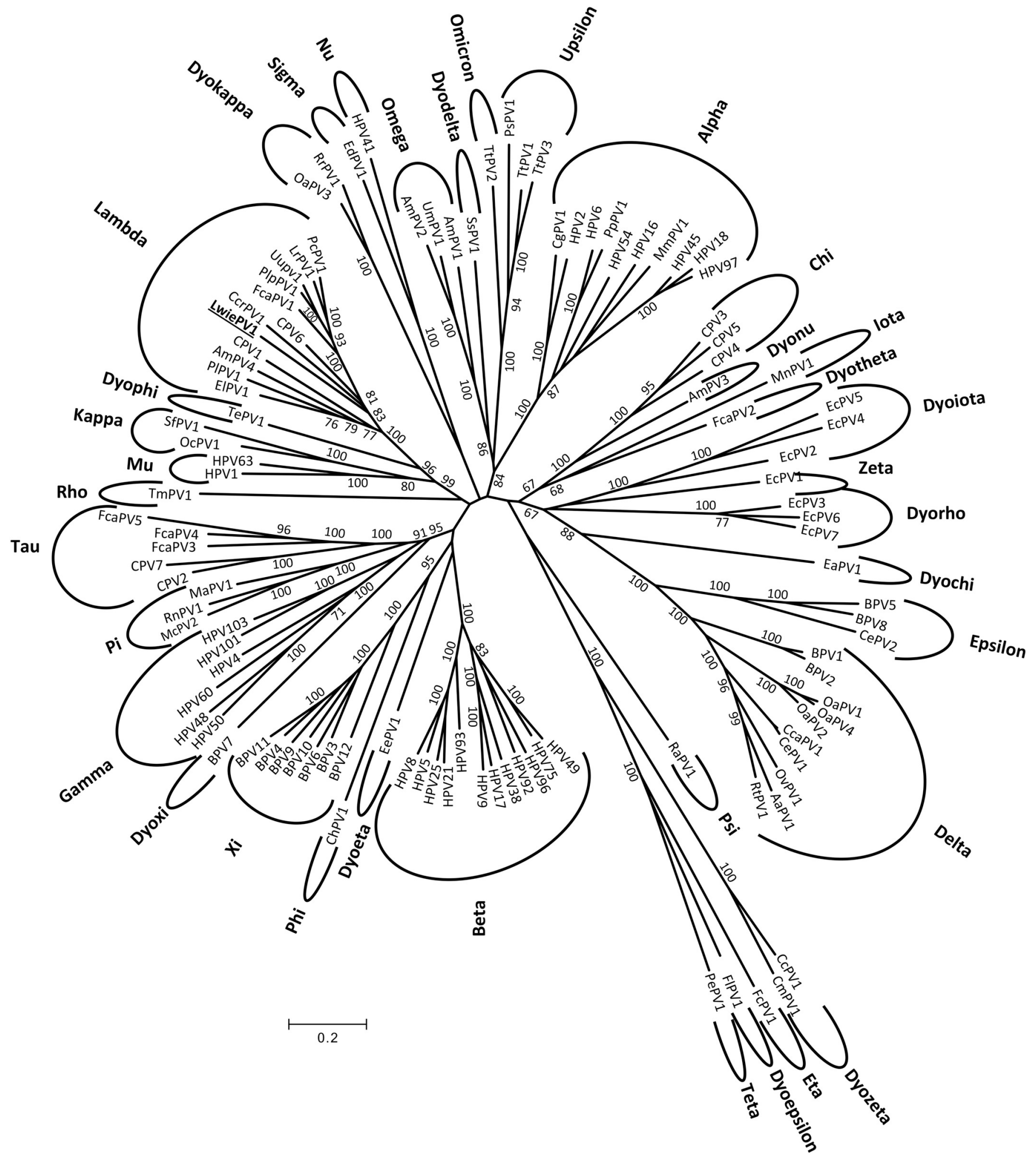

Fig. 2. Maximum likelihood tree including LwiePV1 and 108 PV-types, chosen in this study as representatives of different papillomavirus genera and species. Phylogeny was inferred by an E1-E2-L1 nucleotide sequence alignment. Genera are indicated by Greek letters, according to the PAVE website (http://pave.niaid.nih. gov). Numbers on branches indicate bootstrap values (only values above 60 are shown). Branch lengths are drawn to scale.

sequenced from PV-containing lesions in 5 feline species: the domestic cat (Felis catus), the bobcat (Lynx rufus), the Florida panther (Puma concolor), the Asian lion (Panthera leo persica), and the snow leopard (Panthera uncia); all viral species (FacPV1, PlpPV1, UuPV1, LrPV1 and PcPV1) belonging to Lambda 1. It has been shown (Rector et al., 2007) that Felidae PV phylogenetic tree topology mirrors that of the hosts tree, despite the complex and dynamic Felidae phylogeographic history.

In this paper we report the full genome sequencing of LwiePV1, the first PV in a Leopardus host, the margay (Leopardus wiedii). LwiePV1 full genome sequencing and reconstruction revealed a genomic asset typical of Lambda PV. Evolutionary analyses correctly assigned LwiePV1 to Lambda PV, confirming a monophyletic origin of the PV species 


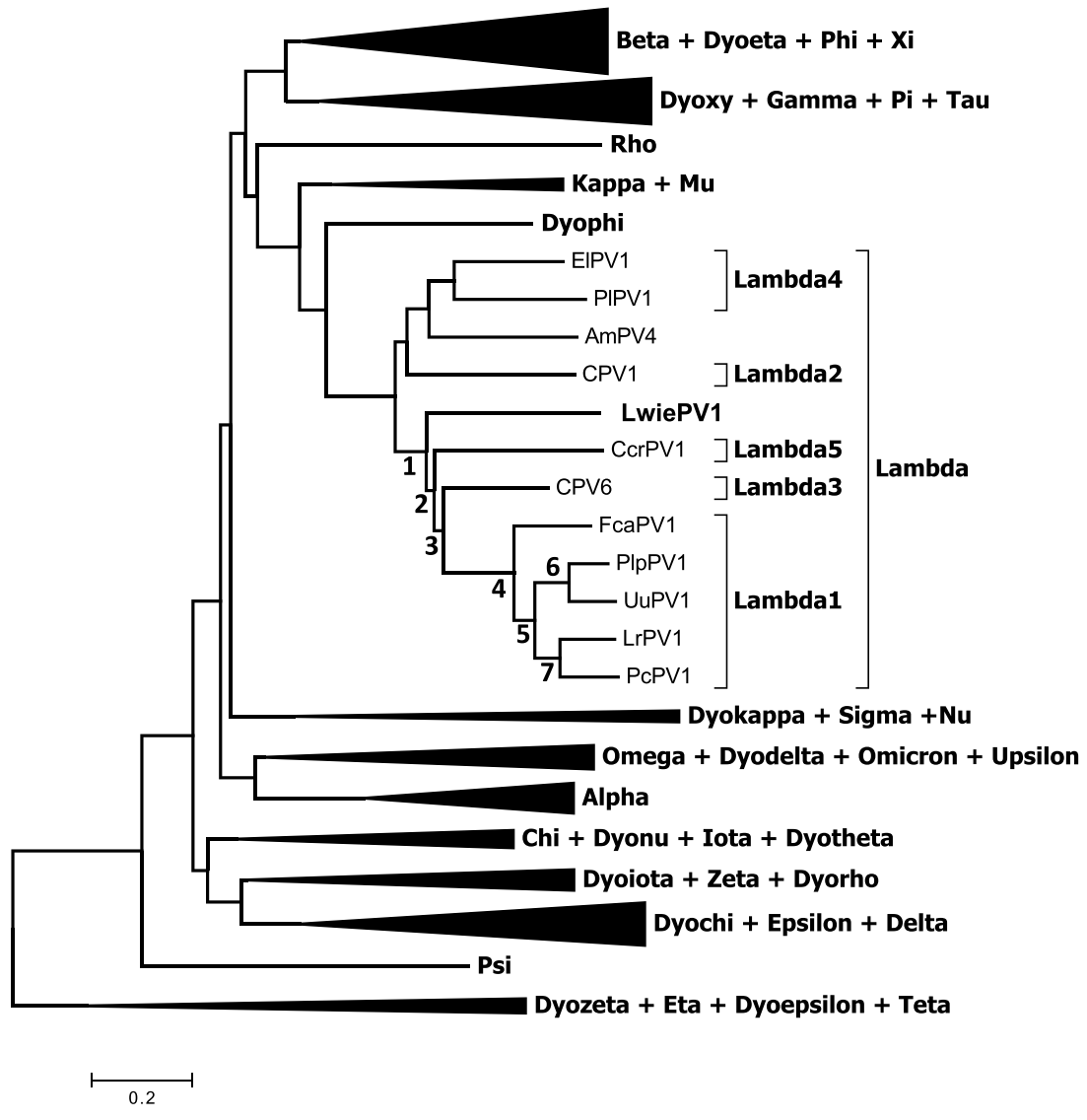

Fig. 3. Maximum likelihood tree showing Lamdapapillomavirus diversity and evolutionary history. PVs of Felidae group in the monophyletic Lambda 1 species, which evolved between about 15 and $8 \mathrm{Myr}$, during the time encompassing the origin of Felidae lineages. LwiePV1 represents an exception to the Lambda 1 rule, and separated as a basal independent branch ancestral to the origin of the canine CPV6 and the hyena CcrPV1. Numbers indicate divergence time of the closer node: $1=31.4 \mathrm{Myr}$ (24.75-46.35); 2 = 30.2 Myr (28.83-44.64); 3 = 27,88 Myr (22-41.2); $4=15.1 \mathrm{Myr}(11.9-22.32) ; 5=12.8 \mathrm{Myr}(10.08-18.9) ;$ 6 = 6.97 Myr (5.5-10.3); 7 = 8,13 Myr (6.42-12). belonging to this genus. Notably, in trees LwiePV1 does not fall in the monophyletic cluster (Lambda 1 species) with the other Felidae PVs, but it emerges as an ancestral, well distinct branch basal to Hyenidae, Canidae and Felidae Lambda Pvs. By including LwiePV1 in phylogenetic trees, Felidae PVs appear therefore as a polyphyletic group, introducing a possible exception to co-divergence and host-dependent evolution during feline PVs evolution. This is confirmed by the tanglegram obtained with Dendroscope, showing LwiePV1 as a clear exception to virus host co-speciation, which is in turn maintained in the other Felidae. Also, tree topology tests support this scenario, and reject the hypothesis of paraphyletic/monophyletic origin of Felidae lambdapapillomaviruses when LwiePV1 is forced to join the other Felidae PVs.

Assuming a constant evolutionary rate for PVs, and according to the overall evolutionary rate of feline PV viral coding genomes, LwiePV1

Papillomavirus

Host
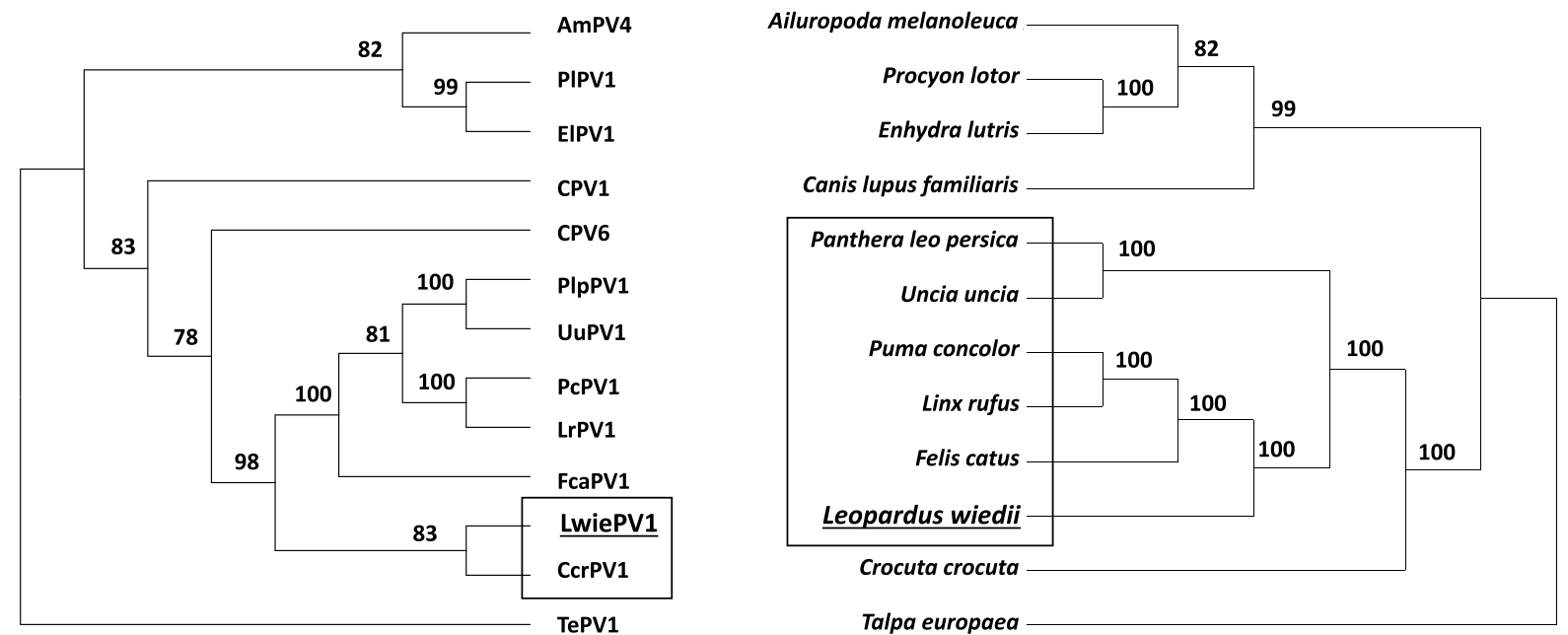

Fig. 4. Tanglegram showing relationships among felid species and their papillomaviruses. For PVs phylogeny (left), a subtree of the full PVs tree based on the E1-E2-L1 nucleotide sequence alignment was used to generate the newick file. Felidae Maximum likelihood tree (right) was based on the entire mitochondrial genome. Bootstrap values are shown for each node along the tree branches. 
A

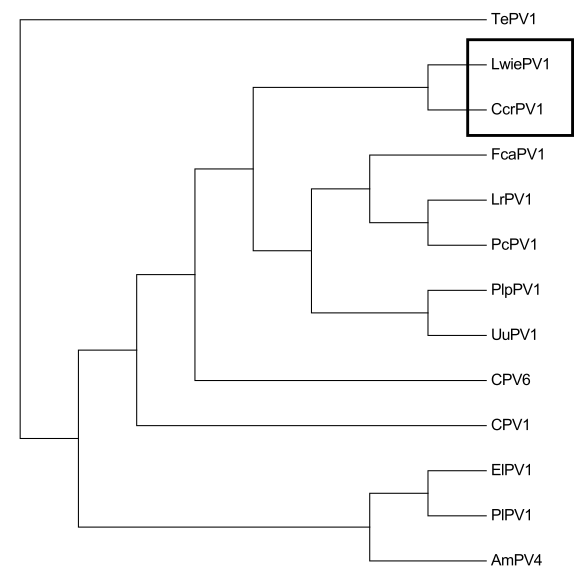

B

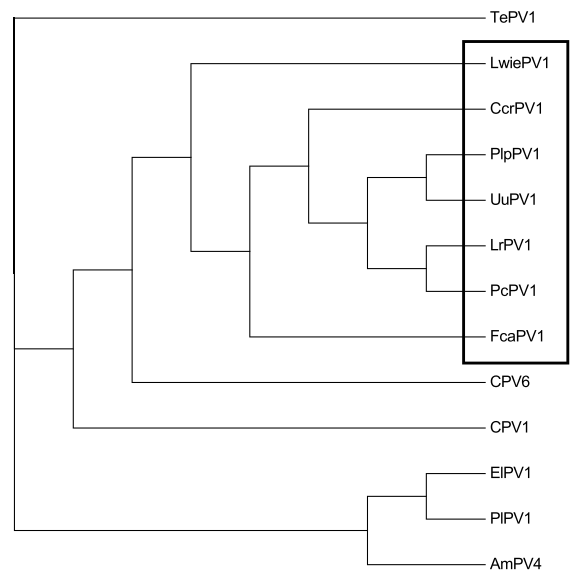

C

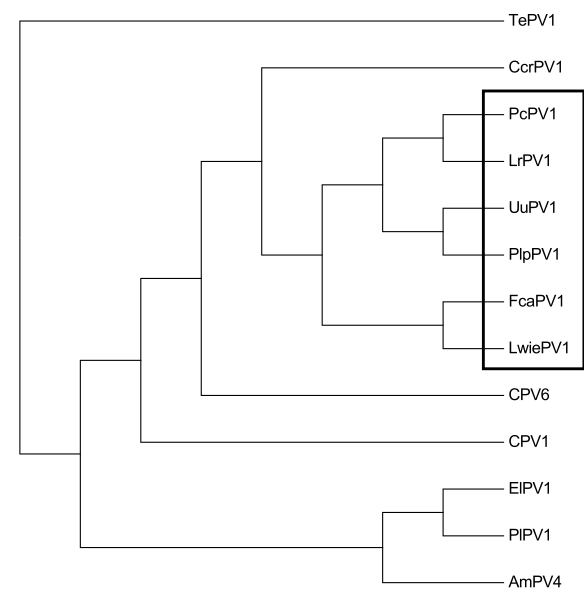

\begin{tabular}{|llllll|}
\hline Tree & logL & deltaL & bp-RELL & c-ELW & p-AU \\
\hline- & & & $1+$ & $1+$ & $1+$ \\
A & -34273.44866 & 0 & 0 & $1.94 e-15-$ & $2.92 e-61-$ \\
C & -34361.60636 & 88.158 & $0-$ & $4.73 e-26-$ & $1.51 e-57-$ \\
\hline
\end{tabular}

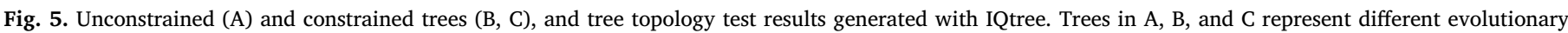

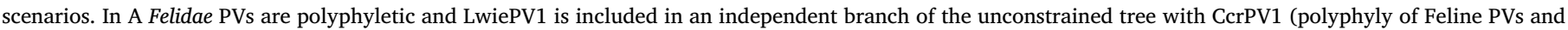

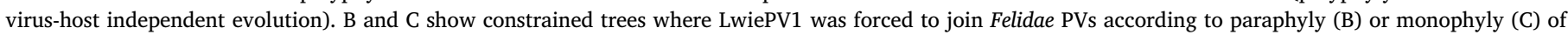

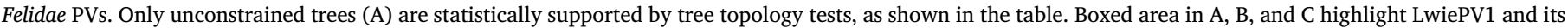
close relatives according to the evolutionary scenario presented.

appeared about $30 \mathrm{Ma}$ (Myr) ago (24-46 Myr), during the time encompassing speciation of the Hyenidae CcrPV1 (30.2 Myr, 28.83-44.64 Myr) and the Canidae CPV6 (27,88 Myr, 22-41.2 Myr), far before the Ocelot lineage originated $8 \mathrm{Myr}$ ago (O'Brien and Johnson, 2007).

Instead, Felidae PVs belonging to the Lambda 1 species show divergence times consistent with the evolution of their respective host, and emerged between about 6 to $15 \mathrm{Myr}$ ago. According to this scenario, observations made by Rector and coworkers (Rector et al., 2007) on feline lambdapapillomaviruses should apply only to the Lambda 1 viruses, which belong to the same ancestral PV lineage, and fulfil the temporal requirement for putative co-divergence. LwiePV1 seems to belong to a more ancient, distinct lineage.

However, the presented trees represent hypotheses that are susceptible to methodological error. As an example, phylogenies based on single genes (L1, E1, or E2, see supplementary material) generate trees with distinct topologies, and E1-based trees are consistent with the host tree, in which LwiePV1 is placed in the feline monophyletic PV branch. However, single genes bootstrap values are low, and the single gene approach is not recommended to infer PV phylogeny.

Also, the discovery of novel feline Lambdapapillomaviruses might modify the evolutionary scenario we pictured, and further research is needed to confirm their phylogenetic relationships.

\section{Conclusions}

The detection and genome characterization of LwiePV1 extends our knowledge on the presence of feline PVs to the Leopardus genus, and represents an attempt to fill the lack of information about PVs infecting other major Felidae lineages. The discovery of novel PV species and hosts is paramount for the correct reconstruction of viral origin and evolution, which is still strongly influenced by the actual fragmentary knowledge of animal PV diversity.

\section{Author contribution}

Gaby Dolz, Roberta Lecis, Antony Solórzano-Morales, Fernando Aguilar-Vargas, Tamara Solórzano-Scott, Roberto Peña, Rosanna Zobba, Gessica Tore, Marco Pittau conducted the research and investigation process, specifically participating to sampling and/or performing the experiments, or data/evidence collection.

Gaby Dolz and Roberta Lecis participated to the preparation of this manuscript.

Gaby Dolz was involved in formulation or evolution of overarching research goals and aims.

Roberta Lecis and Alberto Alberti designed and developed methodology.

Alberto Alberti conceived and supervised the research, elaborated data and prepared this manuscript.

\section{Declaration of Competing Interest}

The authors declare no conflicts of interest associated with the current study.

\section{Appendix A. Supplementary data}

Supplementary data to this article can be found online at https:// doi.org/10.1016/j.meegid.2020.104239.

\section{References}

Alberti, A., Pirino, S., Pintore, F., Addis, M.F., Chessa, B., Cacciotto, C., Cubeddu, T., Anfossi, A., Benenati, G., Coradduzza, E., Lecis, R., Antuofermo, E., Carcangiu, L. Pittau, M., 2010. Ovis aries papillomavirus 3: a prototype of a novel genus in the family Papillomaviridae associated with ovine squamous cell carcinoma. Virology 407, 352-359.

Antonsson, A., McMillan, N.A.J., 2006. Papillomavirus in healthy skin of Australian animals. J. Gen. Virol. 87, 3195-3200. 
Bernard, H.U., Burk, R.D., Chen, Z., van Doorslaer, K., Zur Hausen, H., de Villiers, E.M., 2010. Classification of papillomaviruses (PVs) based on 189 PV types and proposal of taxonomic amendments. Virology 401, 70-79.

Chen, Z., et al., 2009. Genomic diversity and interspecies host infection of $\alpha 12$ Macaca fascicularis papillomaviruses (MfPVs). Virology 393, 304-310.

Da Silva, F.R.C., Cibulski, S.P., Daudt, C., Weber, M.N., Guimarães, L.L., Streck, A.F., Mayer, F.Q., Roehe, P.M., Canal, C.W., 2016 Sep 8. Novel bovine papillomavirus type discovered by rolling-circle amplification coupled with next-generation sequencing. PLoS One 11 (9), e0162345. https://doi.org/10.1371/journal.pone.0162345. eCollection 2016.

De Oliveira, T., Paviolo, A., Schipper, J., Bianchi, R., Payan, E., Carvajal, S.V., 2015. Leopardus wiedii. The IUCN Red List of Threatened Species. (e.T11511A50654216).

De Villiers, E.M., Fauquet, C., Broker, T.R., Bernard, H.U., Zur Hausen, H., 2004. Classification of papillomaviruses. Virology 20, 17-27.

Felsenstein, J., 1985. Confidence limits on phylogenies: an approach using the bootstrap. Evolution 39, 783.

García-Vallvé, S., Alonso, Á., Bravo, I.G., 2005. Papillomaviruses: Different genes have different histories. Trends Microbiol. 13, 514-521.

Gottschling, M., Göker, M., Stamatakis, A., Bininda-Emonds, O.R., Nindl, I., Bravo, I.G. 2011. Quantifying the phylodynamic forces driving papillomavirus evolution. Mol. Biol. Evol. 28, 2101-2113.

Hall, T.A., 1999. BioEdit: a user-friendly biological sequence alignment editor and analysis program for windows 95/98/NT. Nucleic Acids Symp. Ser. 41, 95-98.

Herbst, L.H., Lenz, J., Van Doorslaer, K., Chen, Z., Stacy, B.A., Wellehan, J.F., Manire, C.A., Burk, R.D., 2009. Genomic characterization of two novel reptilian papillomaviruses, Chelonia mydas papillomavirus 1 and Caretta caretta papillomavirus 1 . Virology 383, 131-135.

Huson, D.H., Scornavacca, C., 2012. Dendroscope 3- an interactive viewer for rooted phylogenetic trees and networks. Syst. Biol. 61, 1061-1067.

Isegawa, N., Ohta, M., Shirasawa, H., Tokita, H., Yamaura, A., Simizu, B., 1995. Nucleotide sequence of a canine oral papillomavirus containing a long noncoding region. Int. J. Oncol. 7, 155-159.

Jackson, A.P., 2005. The effect of paralogous lineages on the application of reconciliation analysis by cophylogeny mapping. Syst. Biol. 54, 127-145.

Kalyaanamoorthy, S., Minh, B.Q., Wong, T.K.F., von Haeseler, A., Jermiin, L.S., 2017. ModelFinder: fast model selection for accurate phylogenetic estimates. Nat. Methods $14,587-589$.

Kishino, H., Miyata, T., Hasegawa, M., 1990. Maximum likelihood inference of protein phylogeny and the origin of chloroplasts. J. Mol. Evol. 31, 151-160.

Lange, C.E., Tobler, K., Markau, T., Alhaidari, Z., Bornand, V., Stöckli, R., Trüssel, M., Ackermann, M., Favrot, C., 2009a. Sequence and classification of FdPV2, a papillomavirus isolated from feline Bowenoid in situ carcinomas. Vet. Microbiol. 137, 60-65.

Lange, C.E., Tobler, K., Ackermann, M., Panakova, L., Thoday, K.L., Favrot, C., 2009b Three novel canine papillomaviruses support taxonomic clade formation. J. Gen. Virol. 90, 2615-2621.

Lange, C.E., Favrot, C., Ackermann, M., Gull, J., Vetsch, E., Tobler, K., 2011. Novel snake papillomavirus does not cluster with other non-mammalian papillomaviruses. Virol. J. 8, 436 .

Lange, C.E., Vetsch, E., Ackermann, M., Favrot, C., Tobler, K., 2013. Four novel papillomavirus sequences support a broad diversity among equine papillomaviruses. J. Gen. Virol. 94, 1365-1372.

Le, S.Q., Gascuel, O., 2008. An improved general amino acid replacement matrix. Mol. Biol. Evol. 25, 1307-1320.

Lecis, R., Tore, G., Scagliarini, A., Antuofermo, E., Dedola, C., Cacciotto, C., Dore, G.M., Coradduzza, E., Gallina, L., Battilani, M., Anfossi, A.G., Muzzeddu, M., Chessa, B., Pittau, M., Alberti, A., 2014. Equus asinus papillomavirus (EaPV1) provides new insights into equine papillomavirus diversity. Vet. Microbiol. 170, 213-223.

López-Bueno, A., Mavian, C., Labella, A.M., Castro, D., Borrego, J.J., Alcami, A., Alejo, A., 2016. Concurrence of iridovirus, polyomavirus, and a unique member of a new group of fish papillomaviruses in lymphocystis disease-affected gilthead sea bream. J. Virol. 90, 8768-8779.

Lyal, C.H.C., Stone, A.R., Hawksworth, D.L., 1986. Coevolutionary Relationships of Lice and Their Hosts: A Test of Fahrenholz's Rule, Coevolution and Systematics. Oxford Clarendon Press, pp. 77-91.

Munday, J.S., 2014. Papillomaviruses in felids. Vet. J. 199, 340-347.

Munday, J.S., Dunowska, M., Hills, S.F., Laurie, R.E., 2013. Genomic characterization of Felis catus papillomavirus-3: A novel papillomavirus detected in a feline Bowenoid in situ carcinoma. Vet. Microbiol. 165, 319-325.

Munday, J.S., Dittmer, K.E., Thomson, N.A., Hills, S.F., Laurie, R.E., 2017. Genomic characterisation of Felis catus papillomavirus type 5 with proposed classification within a new papillomavirus genus. Vet. Microbiol. 207, 50-55.

Myers, G., Lu, H., Calef, C., Leitner, T., 1996. Heterogeneity of papillomaviruses. Semin.
Cancer Biol. 7, 349-358.

Nguyen, L.T., Schmidt, H.A., von Haeseler, A., Minh, B.Q., 2015. IQ-TREE: a fast and effective stochastic algorithm for estimating maximum likelihood phylogenies. Mol. Biol. Evol. 32, 268-274.

O’Brien, S.J., Johnson, W.E., 2007. The evolution of cats. Sci. Am. July, 68-75.

Perrière, G., Gouy, M., 1996. WWW-query: an on-line retrieval system for biological sequence banks. Biochimie 78, 364-369.

Rector, A., van Doorslaer, K., Bertelsen, M., Barker, I.K., Olberg, R.A., Lemey, P., Sundberg, J.P., Van Ranst, M., 2005. Isolation and cloning of the raccoon (Procyon lotor) papillomavirus type 1 by using degenerate papillomavirus-specific primers. J. Gen. Virol. 86, 2029-2033.

Rector, A., Van Ranst, M., 2013. Animal papillomaviruses. Virology 445, 213-223.

Rector, A., Lemey, P., Tachezy, R., Mostmans, S., Ghim, S.J., Van Doorslaer, K., Roelke, M., Bush, M., Montali, R.J., Joslin, J., Burk, R.D., Jenson, A.B., Sundberg, J.P., Shapiro, B., Van Ranst, M., 2007. Ancient papillomavirus-host co-speciation in Felidae. Genome Biol. 8, R57.

Scagliarini, A., Gallina, L., Battilani, M., Turrini, F., Savini, F., Lavazza, A., Chiari, M., Coradduzza, E., Peli, A., Erdélyi, K., Alberti, A., 2013. Cervus elaphus papillomavirus (CePV1): new insights on viral evolution in deer. Vet. Microbiol. 165, 252-259.

Shimodaira, H., 2002. An approximately unbiased test of phylogenetic tree selection. Syst. Biol. 51, 492-508.

Stamatakis, A., 2006. RAxML-VI-HPC: maximum likelihood-based phylogenetic analyses with thousands of taxa and mixed models. Bioinformatics 22, 2688-2690.

Stevens, H., Heylen, E., De Keyser, K., Maes, R., Kiupel, M., Wise, A., Nelson, K., Holekamp, K., Engh, A., McKnight, C., Van Ranst, M., Rector, A., 2013. Complete genome sequence of the Crocuta crocuta papillomavirus type 1 (CcrPV1) from a spotted hyena, the first papillomavirus characterized in a member of the Hyaenidae. Genome Announc. https://doi.org/10.1128/genomeA.00062-12.

Strimmer, K., Rambaut, A., 2002. Inferring confidence sets of possibly misspecified gene trees. Proc. Biol. Sci. 22, 137-142.

Tachezy, R., Rector, A., Havelkova, M., Wollants, E., Fiten, P., Opdenakker, G., Jenson, B., Sundberg, J., Van Ranst, M., 2002. Avian papillomaviruses: the parrot Psittacus erithacus papillomavirus (PePV) genome has a unique organization of the early protein region and is phylogenetically related to the chaffinch papillomavirus. BMC Microbiol. 2, 19-28.

Tamura, K., Stecher, G., Peterson, D., Filipski, A., Kumar, S., 2013. MEGA6: molecular evolutionary genetics analysis version 6.0. Mol. Biol. Evol. 30, 2725-2729.

Terai, M., DeSalle, R., Burk, R.D., 2002. Lack of canonical E6 and E7 open reading frames in bird papillomaviruses: Fringilla coelebs papillomavirus and Psittacus erithacus timneh papillomavirus. J. Virol. 76, 10020-11002.

Thompson, J.D., Higgins, D.G., Gibson, T.J., 1994. CLUSTAL W: improving the sensitivity of progressive multiple sequence alignment through sequence weighting, positionspecific gap penalties and weight matrix choice. Nucleic Acids Res. 22, 4673-4680.

Tore, G., Cacciotto, C. Anfossi, A.G., Dore, G.M., Antuofermo, E., Scagliarini, A., Burrai, G.P., Pau, S., Zedda, M.T., Masala, G., Pittau, M., Alberti, A., 2017. Host cell tropism, genome characterization, and evolutionary features of OaPV4, a novel Deltapapillomavirus identified in sheep fibropapilloma. Vet. Microbiol. 204, 151-158.

Van Doorslaer, K., Sidi, A.O., Zanier, K., Rybin, V., Deryckère, F., Rector, A., Burk, R.D., Lienau, E.K., van Ranst, M., Travé, G., 2009. Identification of unusual E6 and E7 proteins within avian papillomaviruses: cellular localization, biophysical characterization, and phylogenetic analysis. J. Virol. 83, 8759-8770.

Van Doorslaer, K., Tan, Q., Xirasagar, S., Bandaru, S., Gopalan, V., Mohamoud, Y., Huyen, Y., McBride, A.A., 2013. The Papillomavirus Episteme: a central resource for papillomavirus sequence data and analysis. Nucleic Acids Res. 41.

Van Ranst, M., Kaplan, J.B., Burk, R.D., 1992. Phylogenetic classification of human papillomaviruses: correlation with clinical manifestations. J. Gen. Virol. 73 , 2653-2660.

Varsani, A., Kraberger, S., Jennings, S., Porzig, E.L., Julian, L., Massaro, M., Pollard, A., Ballard, G., Ainley, D.G., 2014. A novel papillomavirus in Adélie penguin (Pygoscelis adeliae) faeces sampled at the cape crozier colony, Antarctica. J. Gen. Virol. 95, $1352-1365$.

Xia, X., Xie, Z., 2001. DAMBE: software package for data analysis in molecular biology and evolution. J. Hered. 92, 371-373.

Zhang, W., Yang, S., Shan, T., Hou, R., Liu, Z., Li, W., Guo, L., Wang, Y., Chen, P., Wang, X., Feng, F., Wang, H., Chen, C., Shen, Q., Zhou, C., Hua, X., Cui, L., Deng, X., Zhang, Z., Qi, D., Delwart, E., 2017. Virome comparisons in wild-diseased and healthy captive giant pandas. Microbiome 5, 90.

Zhu, W., Dong, J., Shimizu, E., Hatama, S., Kadota, K., Goto, Y., Haga, T., 2012. Characterization of novel bovine papillomavirus type 12 (BPV-12) causing epithelial papilloma. Arch. Virol. 157, 85-91.

Zur Hausen, H., 2009. Papillomaviruses in the causation of human cancers - a brief historical account. Virology 384, 260-265. 